\title{
A novel method of cooling channel optimization for active-cooled structure using the calculus of variations
}

\author{
Xuan $\mathrm{Li}^{1}$, Yang $\mathrm{Lu}^{2}$, Kun $\mathrm{Wu}^{3}$, Xuejun $\mathrm{Fan}^{4}$ \\ Key Laboratory of High Temperature Gas Dynamics, Institute of Mechanics, CAS, Beijing 100190, \\ People's Republic of China
}

School of Engineering Science, University of Chinese Academy of Sciences, Beijing 100049,

People's Republic of China

\begin{abstract}
The optimal design of the regenerative cooling system to improve the comprehensive heat transfer performance has been a long-term interest for researchers. It is essential to develop practical solution to optimize the cooling structure. The novel method of cooling structure optimization based on the calculus of variations has been developed. The average temperature, temperature inhomogeneity and coolant flow pressure loss were chosen as objective functions. The cooling channels can be established depends on geometric boundary conditions and thermal boundary condition of the structure, then the threedimensional simulation of fluid/solid coupling field was calculated to get the temperature distribution of the cooling structure, the loss pressure, etc. Compared with the regualr optimization, the results show that the optimization of cooling structure based on the calculus of variations improves the cooling efficiency: the average temperature decreases, the temperature inhomogeneity drops, although the loss of pressure rises.
\end{abstract}

\section{Nomenclature}

$\begin{array}{ll}P & =\text { pressure } \\ T & =\text { heat flux } \\ q & =\text { heat flux } \\ h t c & =\text { heat transfer coefficient } \\ T w_{1} & =\text { inner wall temperature } \\ T w_{2} & =\text { cooling channel wall temperature } \\ T w_{3} & =\text { cold wall temperature } \\ T_{f} & =\text { coolant temperature } \\ \dot{m} & =\text { mass flowrate of single channel } \\ H_{s} & =\text { total thickness of cooling structure } \\ W_{s} & =\text { total width of cooling structure } \\ m_{s} & =\text { total mass flowrate for cooling structure }\end{array}$

PHD student, Institute of Mechanics (CAS), lixuan@imech.ac.cn

2 Associate Professor, Institute of Mechanics (CAS), luyang@imech.ac.cn.

3 Assistant Professor, Institute of Mechanics (CAS), wukun@imech.ac.cn

${ }^{4}$ Professor, Institute of Mechanics (CAS), xfan@imech.ac.cn, AIAA lifetime member (Corresponding author). 


\section{Introduction}

$\mathrm{A}$ ctively cooling has been an effective thermal management approach to relieve high heat loads for scramjet engines and rockets, in which coolant fuel flows through the channels embedded in the combustor wall to partially cool down the combustor walls before injected into the combustor. To design the cooling channel, the main objective is to maximize the cooling performance with affordable and controllable flow resistance. To this end, the optimization of cooling channel has been a long-term interest to researchers in the scramjet and rocket engine communities[1-9]. Through optimizing the cooling channel, minimize local temperature and thermal stress are attained which enables the combustor to survive more severe thermal environment[10, 11]. Basically, optimal shape design of flow channels can be categorized into three categories: size, shape and topology optimization. For commonly used cooling channels in scramjets or rocket engines, usually size optimizations are employed, in which the width and the height of the cooling channels are adjusted by empirical approaches[12, 13]. Regarding to topology optimization of mechanical structures, the Solid Isotropic Material with Penalization (SIMP) and level set method are prevalent[1,8,14-17]. However, they are regarded to be impractically for scramjet since optimal designs are usually leads to challenges in manufacturing and fabrication.

Fortunately, with the rapid development of 3D-printing technology in the past decade, complex topology and arbitrarily geometries can be realized, which leads the combustor designers to the realm of freedom. Consequently, cooling channel optimizations conforming to the geometric characteristics of the combustor chamber while automatically adapting to the realistic heat flux distribution or temperature distribution become manufacturing and fabrication feasible. Therefore, to optimize the cooling channel for actively cooled structure in scramjets, a novel design philosophy is proposed based on the calculus of variations method in the present study.

The flowrate of the optimization procedure is demonstrated in Fig. 1. First, the design domain and the cooling inlet parameters should be predetermined before calculation. Then an initial thermal environment is obtained by combustor analysis, then temperature, heat flux distributions are provided under the geometry constrains such as measuring port or geometric change. Based on these inputs, cooling channels are generated and optimized by conforming the geometric features while adapting to the temperature or heat flux q distributions subjected to an affordable coolant pressure loss via structure thermal analysis and cooling channel analysis. After several iterations, the optimal cooling channel can be determined which is certainly an improved compromise between cooling performance and hydrodynamic deficiency. It should be noted that the scope of the present work is to investigate and validate the algorithms for determining the layout and diameter of the cooling channels, the coupling between thermal environment and structural thermal analysis, cooling channel analysis merit further investigation.

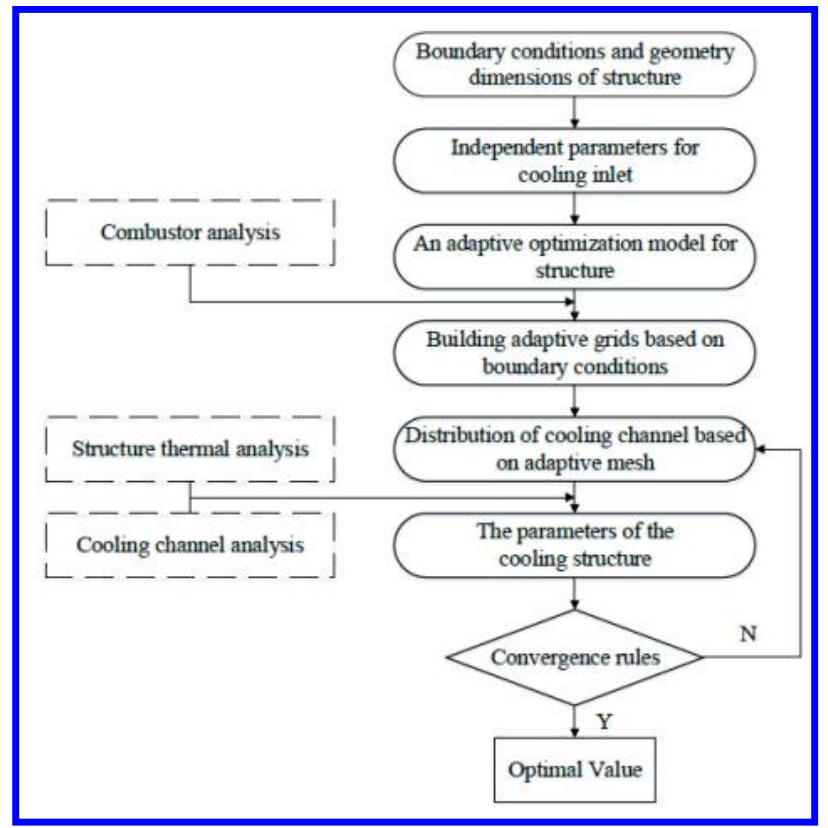

Fig. 1 Flowchart of the optimization procedure 


\section{Numerical Methodology}

\section{A. Adaptive optimization model}

To illustrate the generality of the method, in Fig. 2, here is an example of arbitrary physical space. To quantify the mapping in Fig. 2 between the physical space $(\mathrm{x}, \mathrm{y})$ and the uniformly discretized computational space $(\xi, \eta)$ by the following relations between $(x, y)$ and $(\xi, \eta)$. The inlets of all the cooling channels start from the red line, outlets in the blue line. The $\eta$ line at the constant is the center of the cooling channel and the width of the cooling channel $\mathrm{d}$ is proportional to the grid density. The physical grids density varies with the temperature or $\mathrm{q}$ distribution: the grids become denser where the temperature or $\mathrm{q}$ is larger, then the $\mathrm{d}$ tends to be narrow.
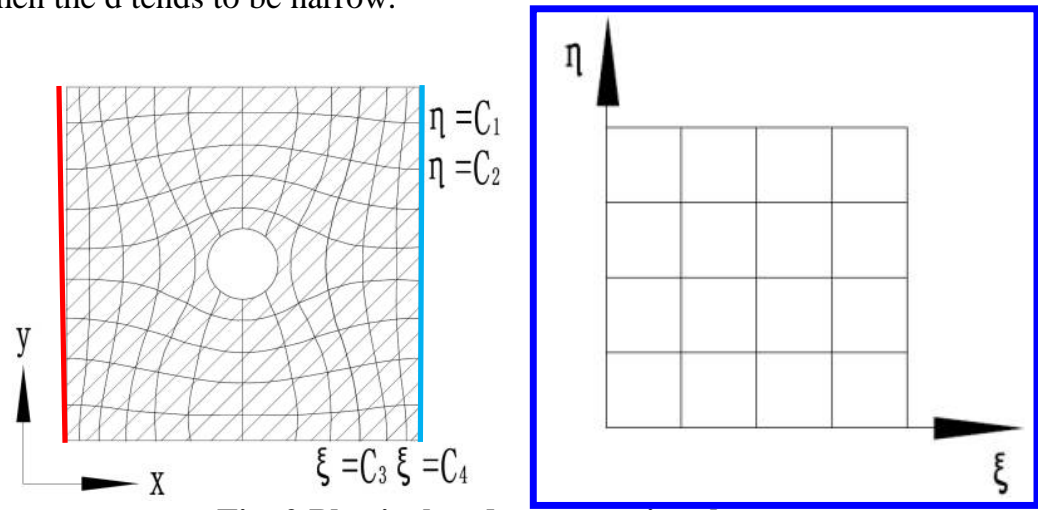

Fig. 2 Physical and computational space

First step, since the potential flow in the domain is incompressible[18], we introduce the complex potential F,

$$
F=\xi+i \eta
$$

F is analytical function. $\xi$ is the potential function $\Phi, \eta$ is the stream function $\Psi$. Its component function $\xi$ and $\eta$ are harmonic, satisfying Laplace's equation:

$$
\left\{\begin{array}{l}
\nabla^{2} \xi=0 \\
\nabla^{2} \eta=0
\end{array}\right.
$$

Where, to interchange the dependent and independent variables by the Laplace's equation, here is

$$
\begin{aligned}
& \alpha x_{\xi \xi}+2 \beta x_{\xi \eta}+\gamma x_{\eta \eta}=0 \\
& \alpha y_{\xi \xi}+2 \beta y_{\xi \eta}+\gamma y_{\eta \eta}=0
\end{aligned}
$$

with coefficients:

$$
\alpha=x_{\eta}^{2}+y_{\eta}^{2}, \quad \beta=-\left(x_{\xi} x_{\eta}+y_{\xi} y_{\eta}\right), \quad \gamma=x_{\xi}^{2}+y_{\xi}^{2}
$$

In this way, the initial mapping is established.

Second step, the developed calculus of variations method is one of the optimizations, where the objective function combining the given function, local orthogonality, grid smoothness is minimized based on the initial mapping[19].

The weighted volume variation, is measured by:

$$
\mathrm{I}_{\mathrm{v}}=\iint_{\mathrm{D}} \omega \mathrm{J} d x d y=\iint_{\mathrm{D}} \omega \mathrm{J}^{2} d \xi d \eta
$$

where $\omega=\omega(x, y)$ is the given function, namely heat flux distribution in the following section.

The global smoothness of the domain is measured by the integral:

$$
\mathrm{I}_{\mathrm{s}}=\iint_{\mathrm{D}}\left[(\nabla \xi)^{2}+(\nabla \eta)^{2}\right] \mathrm{J}^{2} d x d y
$$

The orthogonality of the domain is measured by the Integral:

$$
\begin{gathered}
\mathrm{I}_{\mathrm{o}}=\iint_{\mathrm{D}}(\nabla \xi \cdot \nabla \eta)^{2} \mathrm{~J}^{3} d x d y \\
\mathrm{~J}=x_{\xi} y_{\eta}-x_{\eta} y_{\xi}
\end{gathered}
$$


Here $\mathbf{J}$ is the determinant of the local Jacobian transformation matrix. The integrals above are transformed in the computational space and a linear combination I, of the transformed integrals is minimized. Here, the integral I minimized, listed below:

$$
I=\lambda_{v} I_{v}+\lambda_{s} I_{s}+\lambda_{o} I_{o}
$$

A system of the second order PDE can be solved by the Gauss-Seidel iteration after discretization by central differences in the computational space.

To obtain the Euler equations for the variational problem formulated in the following section, it needs to interchange the dependent and independent variables by the relations:

$$
\xi_{x}=y_{\eta} / J, \xi_{y}=-x_{\eta} / J, \eta_{x}=-y_{\xi} / J, \eta_{y}=x_{\xi} / J
$$

When the variables are interchanged, the smoothness measure can be written as:

$$
\mathrm{I}_{\mathrm{s}}=\iint_{\mathrm{D}}\left[(\nabla \xi)^{2}+(\nabla \eta)^{2}\right] \mathrm{J}^{2} d x d y=\iint_{\mathrm{D}}\left(x_{\xi}^{2}+x_{\eta}^{2}+y_{\xi}^{2}+y_{\eta}^{2}\right) \mathrm{J} d \xi d \eta
$$

For which the corresponding Euler equations are:

$$
\begin{aligned}
& \left(\frac{\partial}{\partial x}-\frac{\partial}{\partial \xi} \frac{\partial}{\partial x_{\xi}}-\frac{\partial}{\partial \eta} \frac{\partial}{\partial x_{\eta}}\right)\left(x_{\xi}^{2}+x_{\eta}^{2}+y_{\xi}^{2}+y_{\eta}^{2}\right) J=0 \\
& \left(\frac{\partial}{\partial y}-\frac{\partial}{\partial \xi} \frac{\partial}{\partial y_{\xi}}-\frac{\partial}{\partial \eta} \frac{\partial}{\partial y_{\eta}}\right)\left(x_{\xi}^{2}+x_{\eta}^{2}+y_{\xi}^{2}+y_{\eta}^{2}\right) J=0
\end{aligned}
$$

Performing the indicated differentiation and collecting coefficients of the highest derivatives yields the equations:

$$
\begin{aligned}
& b_{s 1} x_{\xi \xi}+b_{s 2} x_{\xi \eta}+b_{s 3} x_{\eta \eta}+a_{s 1} y_{\xi \xi}+a_{s 2} y_{\xi \eta}+a_{s 3} y_{\eta \eta}=0 \\
& a_{s 1} x_{\xi \xi}+a_{s 2} x_{\xi \eta}+a_{s 3} x_{\eta \eta}+c_{s 1} y_{\xi \xi}+c_{s 2} y_{\xi \eta}+c_{s 3} y_{\eta \eta}=0
\end{aligned}
$$

where

$$
\begin{gathered}
a_{s 1}=-\left(x_{\xi} x_{\eta}-y_{\xi} y_{\eta}\right), a_{s 2}=x_{\xi}^{2}+y_{\eta}^{2}-x_{\eta}^{2}+y_{\xi}^{2}, a_{s 3}=x_{\xi} x_{\eta}-y_{\xi} y_{\eta} \\
b_{s 1}=J+2 x_{\xi} y_{\eta}, b_{s 2}=-2\left(x_{\xi} y_{\xi}-x_{\eta} y_{\eta}\right), b_{s 3}=J-2 x_{\eta} y_{\xi} \\
c_{s 1}=J-2 x_{\eta} y_{\xi}, c_{s 2}=2\left(x_{\xi} y_{\xi}-x_{\eta} y_{\eta}\right), c_{s 3}=J+2 x_{\xi} y_{\eta}
\end{gathered}
$$

Similarly, the orthogonality measure can be also written as:

$$
\mathrm{I}_{\mathrm{o}}=\iint_{\mathrm{D}}(\nabla \xi \cdot \nabla \eta)^{2} \mathrm{~J}^{3} d x d y=\iint_{\mathrm{D}}\left(x_{\xi} x_{\eta}+y_{\xi} y_{\eta}\right)^{2} d \xi d \eta
$$

For which the Euler equations are:

$$
\begin{aligned}
& b_{o 1} x_{\xi \xi}+b_{o 2} x_{\xi \eta}+b_{o 3} x_{\eta \eta}+a_{o 1} y_{\eta} y_{\xi \xi}+a_{o 2} y_{\xi \eta}+a_{o 3} y_{\eta \eta}=0 \\
& a_{o 1} x_{\xi \xi}+a_{o 2} x_{\xi \eta}+a_{o 3} x_{\eta \eta}+c_{o 1} y_{\eta} y_{\xi \xi}+c_{o 2} y_{\xi \eta}+c_{o 3} y_{\eta \eta}=0
\end{aligned}
$$

with coefficients:

$$
\begin{gathered}
a_{o 1}=x_{\eta} y_{\eta}, a_{o 2}=x_{\xi} y_{\eta}+x_{\eta} y_{\xi}, a_{o 3}=-x_{\xi} y_{\xi} \\
b_{o 1}=x_{\eta}^{2}, b_{o 2}=2\left(2 x_{\xi} x_{\eta}+y_{\xi} y_{\eta}\right), b_{o 3}=x_{\xi}^{2} \\
c_{o 1}=y_{\eta}^{2}, c_{o 2}=2\left(x_{\xi} x_{\eta}+2 y_{\xi} y_{\eta}\right), c_{o 3}=y_{\xi}^{2}
\end{gathered}
$$

The measure of the given function, after interchanging dependent and independent variables, can be written as:

$$
\mathrm{I}_{\mathrm{v}}=\iint_{\mathrm{D}} \omega \mathrm{J} d x d y=\iint_{\mathrm{D}} \omega \mathrm{J}^{2} d \xi d \eta
$$

For which the Euler equations are:

$$
\left(\frac{\partial}{\partial x}-\frac{\partial}{\partial \xi} \frac{\partial}{\partial x_{\xi}}-\frac{\partial}{\partial \eta} \frac{\partial}{\partial x_{\eta}}\right)\left(\omega J^{2}\right)=0
$$




$$
\left(\frac{\partial}{\partial y}-\frac{\partial}{\partial \xi} \frac{\partial}{\partial y_{\xi}}-\frac{\partial}{\partial \eta} \frac{\partial}{\partial y_{\eta}}\right)\left(\omega J^{2}\right)=0
$$

Collecting coefficients of the highest derivatives yields the equations:

$$
\begin{aligned}
& b_{v 1} x_{\xi \xi}+b_{v 2} x_{\xi \eta}+b_{v 3} x_{\eta \eta}+a_{v 1} y_{\xi \xi}+a_{v 2} y_{\xi \eta}+a_{v 3} y_{\eta \eta}=-\frac{J^{2}}{2 \omega} \frac{\partial \omega}{\partial x} \\
& a_{v 1} x_{\xi \xi}+a_{v 2} x_{\xi \eta}+a_{v 3} x_{\eta \eta}+c_{v 1} y_{\xi \xi}+c_{v 2} y_{\xi \eta}+c_{v 3} y_{\eta \eta}=-\frac{J^{2}}{2 \omega} \frac{\partial \omega}{\partial y}
\end{aligned}
$$

where the coefficients are given by:

$$
\begin{gathered}
a_{v 1}=-x_{\eta} y_{\eta}, a_{v 2}=x_{\xi} y_{\eta}+x_{\eta} y_{\xi}, a_{v 3}=-x_{\xi} y_{\xi} \\
b_{v 1}=y_{\eta}^{2}, b_{v 2}=-2 y_{\xi} y_{\eta}, b_{v 3}=y_{\xi}^{2} \\
c_{v 1}=x_{\eta}^{2}, c_{v 2}=-2 x_{\xi} x_{\eta}, c_{v 3}=x_{\xi}^{2}
\end{gathered}
$$

In general, the Euler equations are added together as:

$$
\begin{aligned}
& b_{1} x_{\xi \xi}+b_{2} x_{\xi \eta}+b_{3} x_{\eta \eta}+a_{1} y_{\xi \xi}+a_{2} y_{\xi \eta}+a_{3} y_{\eta \eta}=-\lambda_{v} \frac{J^{2}}{2 \omega} \frac{\partial \omega}{\partial x} \\
& a_{1} x_{\xi \xi}+a_{2} x_{\xi \eta}+a_{3} x_{\eta \eta}+c_{1} y_{\xi \xi}+c_{2} y_{\xi \eta}+c_{3} y_{\eta \eta}=-\lambda_{v} \frac{J^{2}}{2 \omega} \frac{\partial \omega}{\partial y}
\end{aligned}
$$

with coefficients given below:

$$
\begin{aligned}
& a_{i}=\lambda_{v} a_{v i}+\lambda_{s} a_{s i}+\lambda_{o} a_{o i} \\
& b_{i}=\lambda_{v} b_{v i}+\lambda_{s} b_{s i}+\lambda_{o} b_{o i} \\
& c_{i}=\lambda_{v} c_{v i}+\lambda_{s} c_{s i}+\lambda_{o} c_{o i}
\end{aligned}
$$

where $\lambda_{v}, \lambda_{s}, \lambda_{o}$ are positive constants selected under different problems.

To generate a mapping, the PDE should be discretized to solve by iteration. Since $(\xi, \eta)$ are continuous variables which take on integer number at the nodes of the computation grid, a uniformly spaced mapping is formed. The derivatives with respect to the independent variables can be computed on the grid. Where each node of the grid is labelled by the value of $(\xi, \eta)$, namely $(i, j)$, the derivatives at the nodes are discretized using the difference equations:

$$
\begin{gathered}
x_{\xi}=\frac{x_{i+1, j}-x_{i-1, j}}{2 \Delta \xi}, x_{\eta}=\frac{x_{i, j+1}-x_{i, j-1}}{2 \Delta \eta} \\
x_{\xi \xi}=\frac{x_{i+1, j}-2 x_{i, j}+x_{i-1, j}}{(\Delta \xi)^{2}}, x_{\eta \eta}=\frac{x_{i, j+1}-2 x_{i, j}+x_{i, j-1}}{(\Delta \eta)^{2}} \\
x_{\xi \eta}=\frac{x_{i+1, j+1}+x_{i-1, j-1}-x_{i+1, j-1}-x_{i-1, j+1}}{4 \Delta \xi \Delta \eta}
\end{gathered}
$$

Also, the derivatives for y can be discretized similarly.

Third step, the mapping can be established using the calculus of variations method, where the grid can be denser when $\omega$ is bigger. Based on this principle, the width of cooling channels $d$ should be proportional to the $\Delta x(\xi, \eta)$ and $\Delta y(\xi, \eta)$. The $\eta$ line at the constant is the center of the cooling channel and the $d$ is vertical to the $\eta$ line. In the mapping where the $\omega$ increases, the grids become denser, then the hydraulic diameter $\mathrm{d}$ will decrease. In this principle,

$$
\begin{gathered}
d=f(\Delta x(\xi, \eta), \Delta y(\xi, \eta)) \\
d \propto\left\{\sqrt{\left\{[x(\xi+\Delta \xi, \eta)-x(\xi, \eta)]^{2}+[y(\xi+\Delta \xi, \eta)-y(\xi, \eta)]^{2}\right\}}\right\}^{1 / n}
\end{gathered}
$$

Where $\mathrm{n}$ is a variable integer number. In addition, the pressure loss is sensitive to the $\mathrm{d}$ : when the $\mathrm{d}$ becomes narrow, the pressure loss will increase dramatically. The number of cooling channel should be determined by coupling with the thermal analysis method to derive ideal cooling channels distribution.

\section{B. Fluid/solid heat transfer coupling}


Due to the complexity of heat transfer in the cooling channel, it is impossible to take all factors related to the heat transfer into account. Therefore, in order to calculate the flow and the heat transfer in the cooling channel, the following assumptions need to be made:

(1) The outside wall of the cooling channel is adiabatic.

(2) The heat transfer in axial direction is neglected.

(3) $1 \mathrm{Cr} 18 \mathrm{Ni} 9 \mathrm{Ti}$ is employed everywhere in the wall.

(4) The coolant in the cooling channel has no change in physical properties in the radial direction.

(5) The influence of boundary layer is not considered.

Considering the fact that cooling channels are usually long and narrow(Fig. 3), convective heat transfer process of fuel in such cooling channels can be approximated to be one dimensional with decent accuracy.

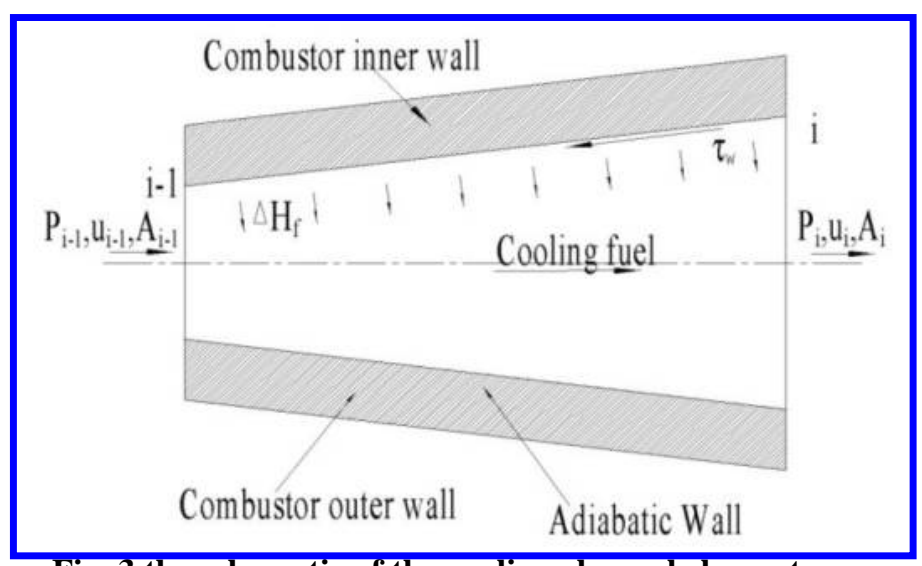

Fig. 3 the schematic of the cooling channel element

According to the governing equations: continuity equation, momentum equation and energy equation, various flow parameters and physical parameters can be achieved by iterations in the calculation process.

Considering that the temperature gradient in flow direction is smaller than that in the cross section, the heat conduction between adjacent sections can be neglected when enough cross sections are divided along the flow direction. In this way, the problem can be transformed to a collection of two-dimensional heat conduction reasonably, which can reduce the cost of calculation. Benefiting from this treatment, the calculation can be programmed in parallel. The two-dimensional structure temperature distribution of cooling structure is achieved via conservative method [20].

\section{Optimization of the cooling structure}

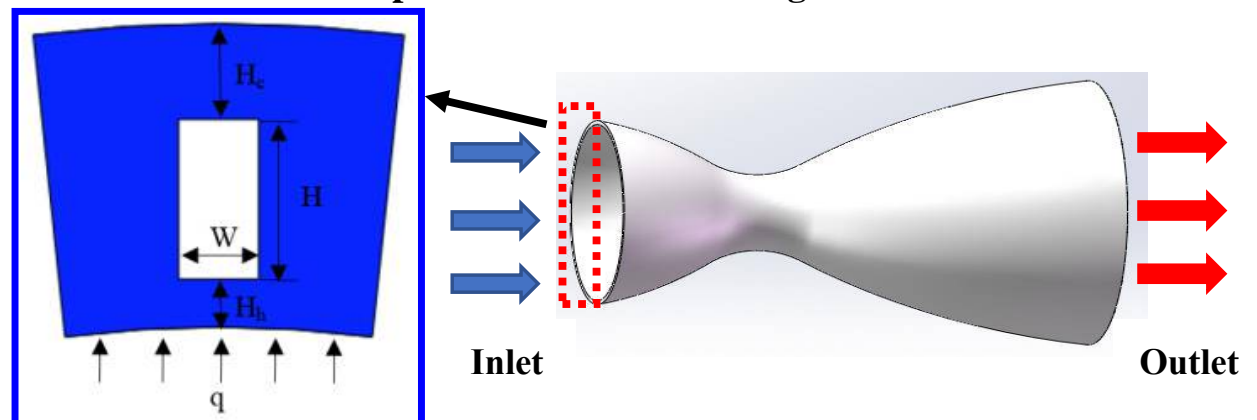

Fig. 4 The geometry of a single channel and schematic diagram of design domain

It can be seen from Fig. 4, the configuration of the cooling structure including cooling inlet, cooling outlet and geometry structure with convergence and divergence. The target is to obtain optimal cooling channels distribution between the inlet and outlet under the constraints. The schematic drawing of a cross section of cooling structure for analysis is given in Fig. 4, among which, $\delta$ is the rib thickness, $\mathrm{W}$ is the width of the cooling channel, $\mathrm{H}$ is the depth of cooling channel, $\mathrm{H}_{\mathrm{c}}$ is the cold side wall thickness, $\mathrm{H}_{\mathrm{h}}$ is the hot side wall thickness, $\mathrm{Tw}_{1}$ represents the inner wall temperature, $\mathrm{Tw}_{2}$ represents the cooling channel wall temperature, $\mathrm{Tw}_{3}$ represents the outside wall temperature. The inner wall is subjected to the heat flux of high temperature gas and outside wall is considered to be adiabatic. Due to the same hot environment of every channel, a single cooling channel can be taken as the research object to be optimized. Since the cooling structure is fixed, the parameters should satisfy:

$$
H_{s}=H_{h}+H+H_{c}
$$




$$
\begin{gathered}
N(W+\delta)=W_{s}=\text { Const } \\
N \dot{m}=m_{s}=\text { Const }
\end{gathered}
$$

$\mathrm{H}_{\mathrm{s}}$ is the total thickness of the cooling structure wall, $\mathrm{W}_{\mathrm{s}}$ is total width of the cooling structure wall, $\mathrm{m}_{\mathrm{s}}$ is the total mass flowrate, $\mathrm{N}$ is the number of cooling channel.

As is known, the heat flux in the combustor usually reaches a few megawatts. Hence, the heat flux imposed on the cooling structure is depicted in Fig. 5. To improve the accuracy of the simulations, all available details were demonstrated in the Table.1.

Table.1 Boundary Conditions

\begin{tabular}{|c|c|c|c|}
\hline Inlet mass flowrate & Inlet Pressure & Inlet Temperature & Length \\
\hline $1 \mathrm{~kg} / \mathrm{s}$ & $60 \mathrm{bar}$ & $300 \mathrm{~K}$ & $270 \mathrm{~mm}$ \\
\hline
\end{tabular}

The shape and the distribution of the cooling channels are formulated via the method described above. Whereas the cooling channel can be generated under this way, the number of the cooling channel still needs to be chosen.

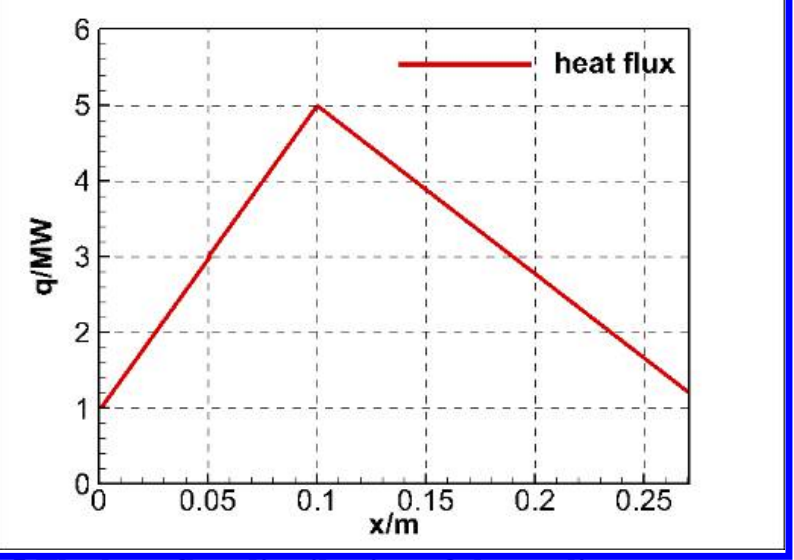

Fig. 5 The heat flux distribution of the cooling structure

The cooling structure is made of $1 \mathrm{Cr} 18 \mathrm{Ni}$ Ti with the temperature limit of $1473 \mathrm{~K}$. The density and specific heat capacity can be treated as constants, and the thermal conductivity is treated as a linear function of temperature:

$$
\lambda=0.01525 \mathrm{~T}+10.6(\mathrm{~W} / \mathrm{mK})
$$

The RP-1 kerosene serves as the coolant and the five-component model is adopted, then the thermal properties of kerosene at the required pressure and temperature can be calculated according to the NIST Supertrapp thermal properties calculation program.

\section{Structure optimization of cooling channel}

By virtue of the optimization method described above, the cooling structure optimization objective function can be demonstrated as following:

$$
\left\{\begin{array}{cl}
\text { find } & Y=\left(y_{1}, y_{2}, \cdots y_{n}\right) \\
\min & F_{0}=w_{1} A+w_{2} B+w_{3} C \\
\text { s.t. } & g_{i}(Y) \leq 0, R_{j}(Y)=0
\end{array}\right.
$$

The objective function can be chosen in many ways. Here, the minimum of average temperature, temperature inhomogeneity and loss of flow pressure is treated as the objective function. Hence,

$$
F_{0}=w_{1} A+w_{2} B+w_{3} C
$$

Among which,

$$
\begin{gathered}
\mathrm{A}=\frac{1}{N_{s}} \sum_{i=1}^{N_{s}} T_{i}, \mathrm{~B}=\frac{1}{V_{s}} \int_{\Omega_{s}}(T-\bar{T})^{2} d \Omega_{s} \\
V_{s}=\int_{\Omega_{s}} d \Omega_{s}, \bar{T}=\frac{1}{V_{s}} \int_{\Omega_{s}} T d \Omega_{s}, \mathrm{C}=\Delta \mathrm{p}
\end{gathered}
$$

Firstly, the average temperature of cooling structure should not be too high in the practical application, otherwise the temperature will exceed the tolerance limit of the material; the temperature inhomogeneity 
should not be too large, or the temperature stress will affect the lifetime of the structure; the loss of flow pressure should be too large, or the additional burden will be added to the system. Considering the processing, the rib thickness should not be less than $1 \mathrm{~mm}$.

In order to facilitate the multi-objective analysis, the objective function is normalized. For the optimization of cooling structure, the objective function can be described as:

$$
F_{0}=w_{1} A / A_{\max }+w_{2} B / B_{\max }+w_{3} C / C_{\max }
$$

Among them, $\mathrm{A}_{\max }, \mathrm{B}_{\max }, \mathrm{C}_{\max }$ are the maximum values of each factor in the range, the weight coefficients $\omega_{1}, \omega_{2}$ and $\omega_{3}$ are selected according to different conditions. Here, there are four cases to be optimized: the specific weight coefficients are set in Table.2, the case1 considers the influence of various factors on average; the case 2 focuses on the decrease of average temperature; the case 3 eyes on the reduction of temperature inhomogeneity; the case 4 emphasizes on the reduction of flow pressure loss.

Table.2 Different weights for different cases

\begin{tabular}{|c|c|c|c|}
\hline Case & $\boldsymbol{\omega}_{\mathbf{1}}$ & $\boldsymbol{\omega}_{\mathbf{2}}$ & $\boldsymbol{\omega}_{\mathbf{3}}$ \\
\hline 1 & 0.33 & 0.33 & 0.33 \\
\hline 2 & 0.6 & 0.2 & 0.2 \\
\hline 3 & 0.2 & 0.6 & 0.2 \\
\hline 4 & 0.2 & 0.2 & 0.6 \\
\hline
\end{tabular}

For the sake of the ultimate goal, the factor $\mathrm{n}$ and the number of cooling channels can be adjusted to obtain the optimization structure. In all the experimental results computed, the following parameters are assumed as: $\lambda_{v}=100, \lambda_{0}=10, \lambda_{\mathrm{s}}=10$.

\section{Results and Discussions}

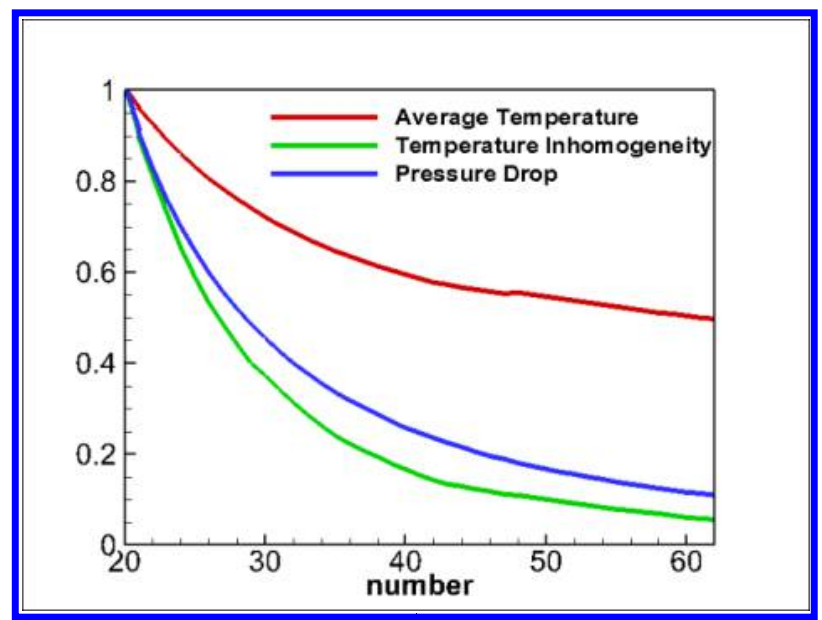

Fig. 6 The variation of variables with number of conling channels

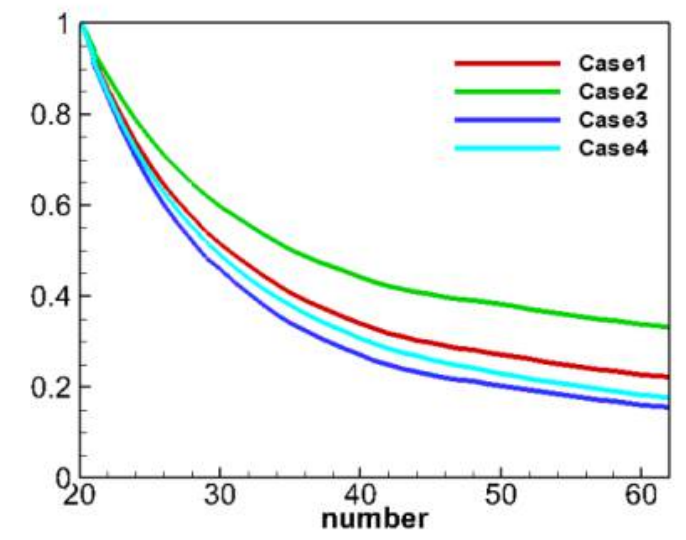

Fig. 7 The variation of different cases with number of cooling channels

In the view of the Fig. 6, with the growth of cooling channel number, the average temperature of cooling structure decreases. Until the number of cooling channel reaches a certain number, the average temperature tends to be stable. Furthermore, when the number of cooling channels increases, the temperature inhomogeneity drops rapidly and then drops slowly. The loss of flow pressure reduces with 
the increase of cooling channel number. In addition, all the factors decrease dramatically with the rise of cooling channel number when the number is in less quantity, then decrease slowly with that in high quantity.

For the four cases mentioned above, it shows that the objective function becomes smaller when the number of cooling passage grows (Fig. 7). It can be found that for all the cases, the minimum objective function locates in the cooling channel number 62 ;

To discuss the details about case 3 , it is concluded that when the number arrives at 62 , the minimum objective function can be achieved. The average temperature of cooling channel is $656 \mathrm{~K}$, the temperature inhomogeneity is $8359 \mathrm{~K}^{2}$. After the calculation, the width of cooling channel not only depends on the heat flux but also on the geometric boundary condition based on the principle. As a consequence, a combination of heat flux and geometric boundary condition, the width of the channel varies in the Fig. 8.

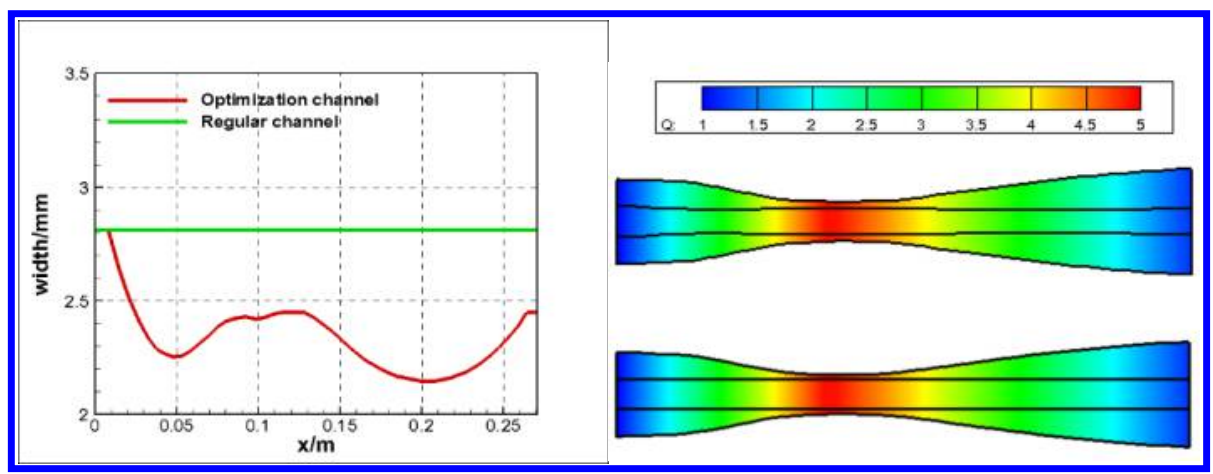

Fig. 8 The width of cooling channel in the structure

In the regular cooling channel design, the cooling channel is generally designed as uniform rectangular structure. It shows that the average temperature of cooling channel is $672 \mathrm{~K}$, the temperature inhomogeneity is $8854 \mathrm{~K}^{2}$ when the width of the regular cooling channel is equal to the widest place in the optimization cooling channel.

Table.3 Comparison of optimization channel and regular channel

\begin{tabular}{|l|c|c|c|}
\hline & $\begin{array}{c}\text { Average } \\
\text { Temperature } \\
/ \mathbf{K}\end{array}$ & $\begin{array}{c}\text { Temperature } \\
\text { Unevenness } \\
/ \mathbf{K}^{\mathbf{2}}\end{array}$ & $\begin{array}{c}\text { Pressure } \\
\text { Drop } \\
\text { /bar }\end{array}$ \\
\hline Optimal & 656 & 8359 & 1.436 \\
\hline Regular & 672 & 8854 & 0.92 \\
\hline
\end{tabular}

The results of optimization channel and regular channel are compared in the Table.3, which reveals that the average temperature decreases from $672 \mathrm{~K}$ to $656 \mathrm{~K}$, the temperature inhomogeneity declines from $8854 \mathrm{~K}^{2}$ to $8349 \mathrm{~K}^{2}$, while the loss of pressure increases from $0.92 \mathrm{bar}$ to $1.436 \mathrm{bar}$ when the regular channel turns into-ontimization channel

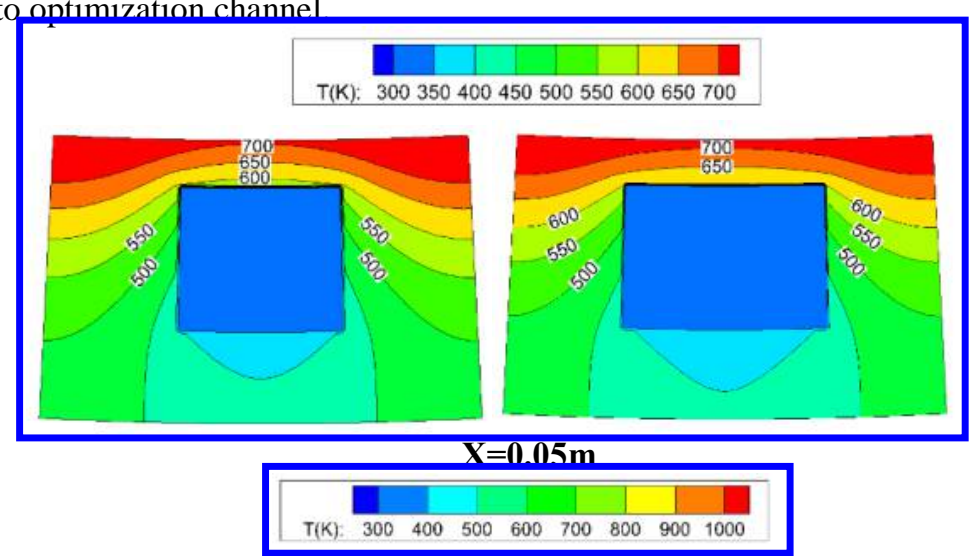




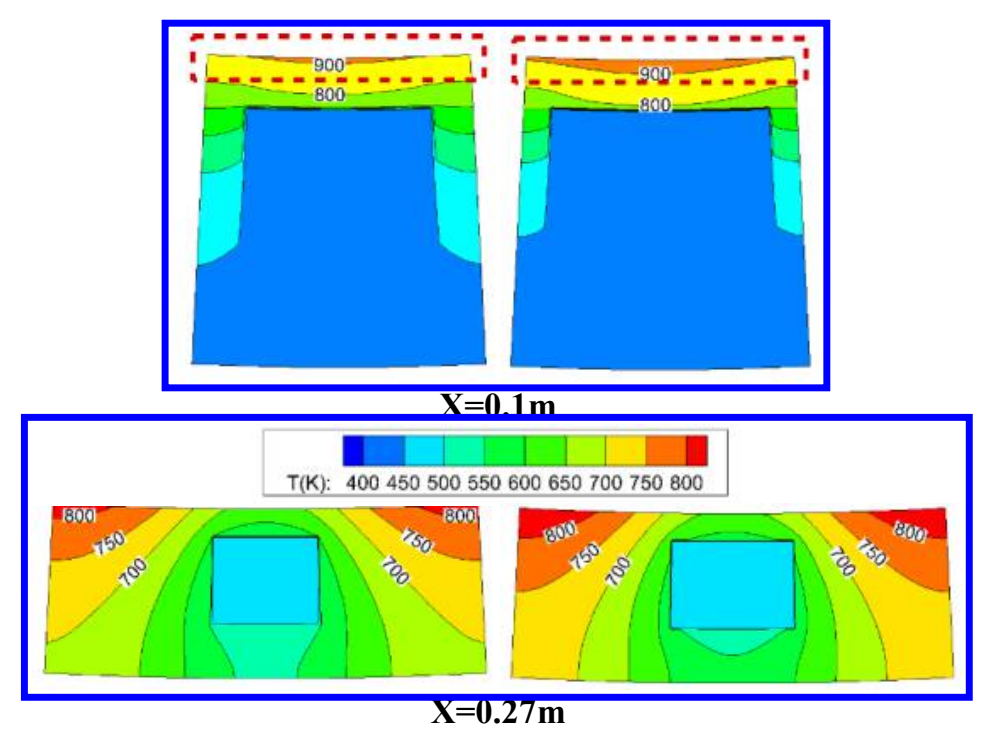

Fig. 9 (Left)The cross-section temperature diagram of optimization channel

(Right) The cross-section temperature diagram of regular channel

The temperature of cross sections is analyzed here: the temperature of cross sections in regular channel are contrast with that in optimization channel (Fig. 9). It is found that the width of the optimization channel is narrower than that of the regular channel and the high temperature zone of optimization channel is smaller than that of regular channel. Take the place where the highest heat flux locates as example, the zone of the temperature above $900 \mathrm{~K}$ in optimization channel is less than the same zone in regular channel obviously. In addition, the $\mathrm{Tw}_{1}$ of regular channel is $927 \mathrm{~K}$ and the $\mathrm{Tw}_{1}$ of optimization channel is $903 \mathrm{~K}$, the difference between them is $24 \mathrm{~K}$. It is easy to say the optimization channel can bring the benefit indeed.

In specific analysis, the velocity in optimization channel is larger than that in regular channel apparently (Fig. 10). This is the reason why the temperature of cross section in optimization channel is lower than that in regular channel. Due to higher velocity in optimization channel, the heat transfer coefficient is larger than that in regular channel, which means in the same hot environment, the coolant can take more heat away. The velocity differences can be as high as 30 percent, which implies the heat transfer improves much. The heat transfer coefficient in optimization channel can be as high as $17000 \mathrm{~W} / \mathrm{m}^{2} \mathrm{~K}$, while that in regular channel is $14300 \mathrm{~W} / \mathrm{m}^{2} \mathrm{~K}$ (Fig 11)

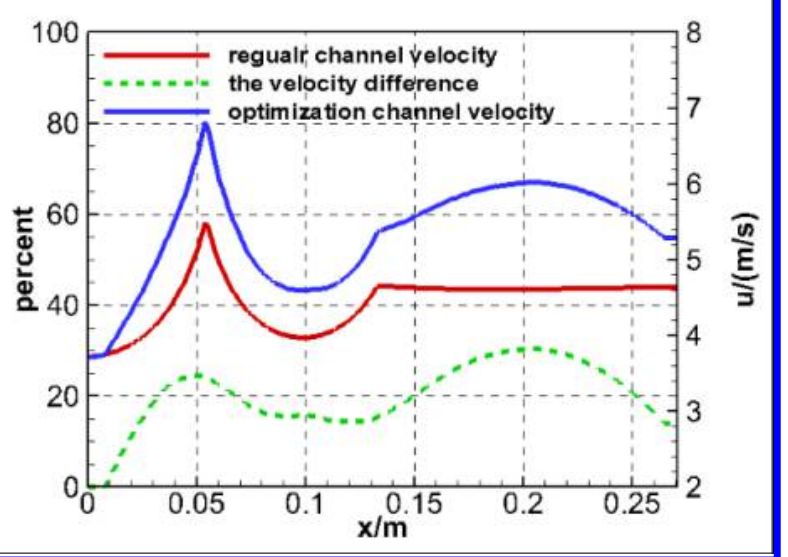

Fig. 10 Comparison of flow velocity in optimization channel and regular channel 


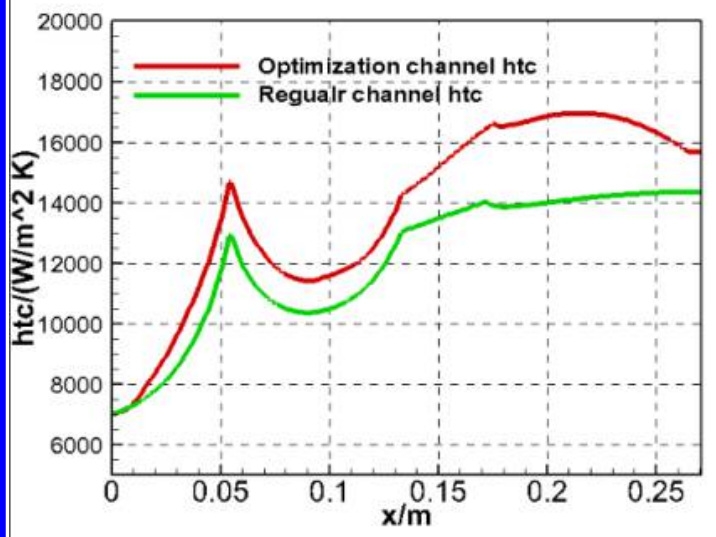

Fig. 11 Comparison of heat transfer coefficient in optimization channel and regular channel

By virtue of the high velocity in optimization channel, the $\mathrm{Tw}_{1}$ of optimization channel is lower than that in regular channel, which can be seen in Fig. 12. The $\mathrm{Tw}_{1}$ in the highest flux place drops from $927 \mathrm{~K}$ to $903 \mathrm{~K}$ when the channel is optimized.

However, some disadvantages appear when the regular channel turns into optimization channel: the loss of pressure climbs from $0.92 \mathrm{bar}$ to $1.436 \mathrm{bar}$ (Fig. 13). Although the width of channel changes a lot from regular to optimization the conlant temnerature keeps still (Fio 14)

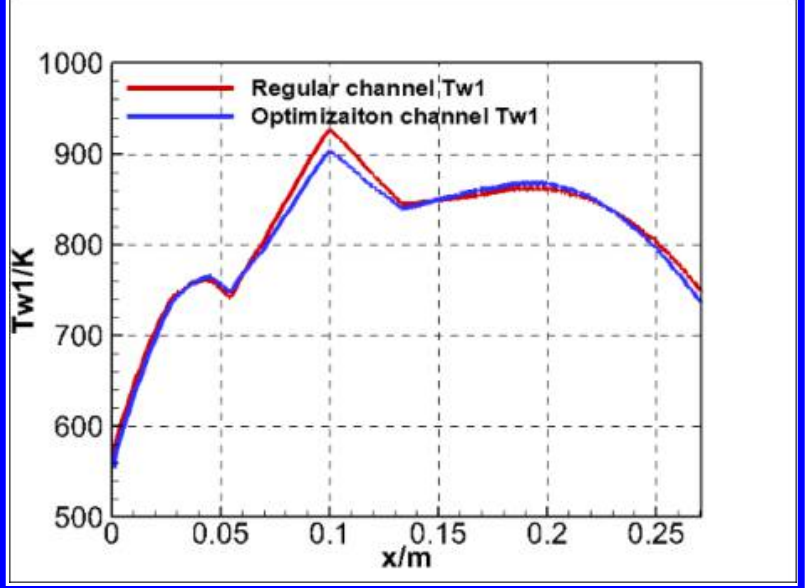

Fig. 12 Comparisonof inner wall femperafure in optimization channeland regular channel

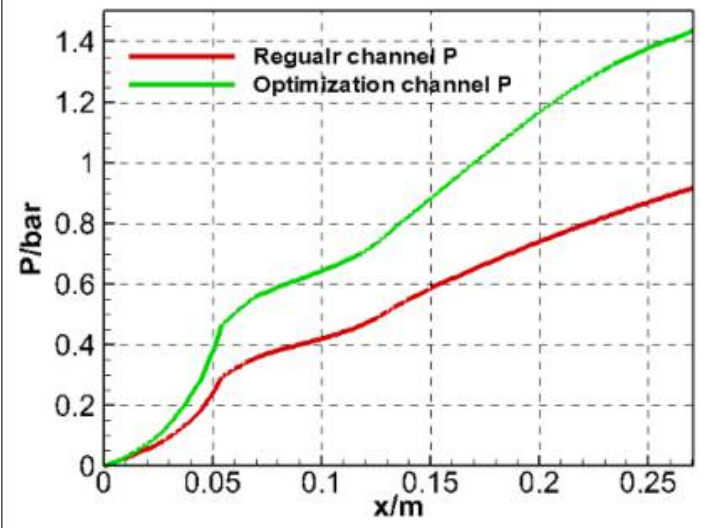

Fig. 13 Comparison of flow pressure loss in optimization channel and regular channel 


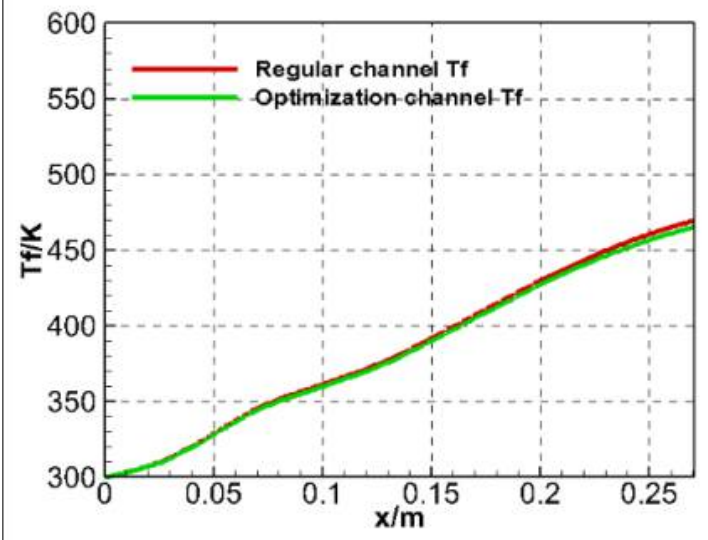

Fig. 14 Comparison of coolant temperature in optimization channel and regular channel

\section{Conclusion and future work}

To develop the optimization method based on the calculus of variations, the cooling structure can be designed more effective than regular design. Based on the optimization method developed here, the cooling channel not only depends on the shape of the structure but also on the heat flux. In a combination of the geometric boundary condition and thermal boundary condition, the cooling channel can be established. By this method, the heat transfer coefficient is larger than that in regular channel, which implies that the optimization channel can take more heat away. The resulting average temperature and temperature inhomogeneity decrease, while the loss of pressure lifts.

In the future, to research the optimization method based on the calculus of variations in depth is a necessary topic. For the novel method, the effect of different parameters determining the efficiency of the cooling structure should be studied. More work should be done to find more effective cooling structure required for improving the cooling efficiency.

\section{References}

1V.J. Challis, J.K. Guest, "Level set topology optimization of fluids in Stokes flow", International Journal for Numerical Methods in Engineering Vol. 79, No. 10,2009, pp. 1284-1308.

${ }^{2}$ X. Chen, "Topology optimization of microfluidics - A review", Microchemical Journal Vol. 127, No. 2016, pp. 52-61.

${ }^{3}$ C. Dapogny, P. Frey, F. Omnès, Y. Privat, "Geometrical shape optimization in fluid mechanics using FreeFem++", Structural and Multidisciplinary Optimization Vol. 58, No. 6,2018, pp. 2761-2788.

${ }^{4}$ Y. Deng, Z. Liu, Y. Liu, Y. Wu, "Combination of topology optimization and optimal control method", Journal of Computational Physics Vol. 257, No. 2014, pp. 374-399.

${ }^{5}$ X. Duan, F. Li, "Material distribution resembled level set method for optimal shape design of Stokes flow", Applied Mathematics and Computation Vol. 266, No. 2015, pp. 21-30.

${ }^{6}$ X. Duan, Y. Ma, R. Zhang, "Optimal shape control of fluid flow using variational level set method", Physics Letters A Vol. 372, No. 9,2008, pp. 1374-1379.

${ }^{7}$ X.-B. Duan, Y.-C. Ma, R. Zhang, "Shape-topology optimization of stokes flow via variational level set method", Applied Mathematics and Computation Vol. 202, No. 1,2008, pp. 200-209.

${ }^{8}$ X.-B. Duan, Y.-C. Ma, R. Zhang, "Shape-topology optimization for Navier-Stokes problem using variational level set method", Journal of Computational and Applied Mathematics Vol. 222, No. 2,2008, pp. 487-499.

$\checkmark{ }^{9}$ J.R.L. Koch, E.M. Papoutsis-Kiachagias, K.C. Giannakoglou, "Transition from adjoint level set topology to shape optimization for 2D fluid mechanics", Computers \& Fluids Vol. 150, No. 2017, pp. 123-138

${ }^{10}$ T. Jing, G. He, B.B. Lin, W. Li, F. Qin, Y. Liu, "Thermal analysis of RBCC engine at ejector, ramjet and scramjet modes", 14th International Energy Conversion Engineering Conference, 2016.

${ }^{11}$ F. Zhong, T. Chen, X. Fan, L. Chen, X. Chang, "Three-Dimensional Heat Transfer Analysis and Optimized Design of Actively Cooled Strut for Scramjet Applications", 48th AIAA/ASME/SAE/ASEE Joint Propulsion Conference \&amp; Exhibit, 2012.

${ }^{12}$ Y. Deng, P. Zhang, Y. Liu, Y. Wu, Z. Liu, "Optimization of unsteady incompressible Navier-Stokes flows using variational level set method", International Journal for Numerical Methods in Fluids Vol. 71, No. 12,2013, pp. 14751493.

${ }^{13}$ K.R. Saviers, R. Ranjan, R. Mahmoudi, "Design and validation of topology optimized heat exchangers", AIAA Scitech 2019 Forum, 2019.

${ }^{14}$ B. Zhang, X. Liu, "Topology optimization study of arterial bypass configurations using the level set method", Structural and Multidisciplinary Optimization Vol. 51, No. 3,2014, pp. 773-798.

${ }^{15}$ S. Zhou, Q. Li, "A variational level set method for the topology optimization of steady-state Navier-Stokes flow", 
Journal of Computational Physics Vol. 227, No. 24,2008, pp. 10178-10195.

${ }^{16} \mathrm{~J}$. Carlile, R. Quentmeyer, "An experimental investigation of high-aspect-ratio cooling passages", 28th Joint Propulsion Conference and Exhibit, 1992.

${ }^{17} \mathrm{Q}$. Wang, F. Wu, M. Zeng, L. Luo, J. Sun, "Numerical simulation and optimization on heat transfer and fluid flow in cooling channel of liquid rocket engine thrust chamber", Engineering Computations Vol. 23, No. 8,2006, pp. 907 921.

${ }^{18}$ K. Sato, "Complex Potential and Differentiation", Complex Analysis for Practical Engineering, 2015, pp. 19-79.

${ }^{19}$ Richard Carcaillet, George S. Dulikravich and Stephen R. Kennon, "generation of solution-adaptive computational grids using optimation", Computer Methods In Applied Mechanics And Engineering, Vol. 57,1986, pp. 279-295.

${ }^{20}$ Thornton E A. A Finite Element Program for Steady-State Thermal Analysis of Convectively Cooled Structure[R]. NASA-CR-145069. 